Received: 20 June 2018

Accepted: 26 February 2019

Published online: 06 May 2019

\section{Long DCL4-substrate dsRNAs efficiently induce RNA interference in plant cells}

\author{
Sayaka Kakiyama ${ }^{1}$, Midori Tabara $\mathbb{D}^{1}$, Yuki Nishibori ${ }^{1}$, Hiromitsu Moriyama ${ }^{1} \&$ \\ Toshiyuki Fukuhara' ${ }^{1,2}$
}

RNA interference (RNAi) is induced by the direct transfer of double-stranded RNAs (dsRNAs) into protoplasts prepared from Arabidopsis thaliana seedlings. In this protoplast RNAi system, we compared the efficacies of various-sized dsRNAs (between 21 and 139 nucleotides [nt]) for inducing RNAi and assessed the dsRNA-cleaving activities of Dicer-like 3 (DCL3) and 4 (DCL4). After the direct transfer of dsRNAs into protoplasts, cleaved RNA products of $21 \mathrm{nt}$ were detected from long 130- or 500-nt dsRNAs by DCL4 but not from 37-nt dsRNAs. These results indicate that DCL4 preferentially cleaves long dsRNAs in protoplasts, consistent with our previous biochemical data regarding the substrate specificity of DCL4. Direct transfer of long dsRNAs of approximately $130 \mathrm{nt}$ into protoplasts induces RNAi much more effectively (by approximately 60- to 400-fold) than direct transfer of short 37-nt dsRNAs. Although transfer of 21-nt dsRNAs into protoplasts induced RNAi without DCL4 activity, the induction of RNAi was less effective (by approximately 0.01 -fold) compared with long dsRNAs. These results indicate that cleavage of long dsRNAs exceeding $100 \mathrm{nt}$ by DCL4 into 21-nt dsRNAs is essential for efficient induction of RNAi in plant cells.

RNA interference (RNAi) is the process of sequence-specific post-transcriptional gene silencing (PTGS) triggered by double-stranded RNAs (dsRNAs) ${ }^{1,2}$. In the RNAi pathway, a Dicer endoribonuclease cleaves long dsRNAs into small interfering RNAs (siRNAs) ${ }^{3}$, which then associate with the protein Argonaute (AGO) $)^{4}$. siRNA-loaded AGO binds specific mRNAs with sequence-complementarity, cleaving them or inhibiting their translation ${ }^{5}$.

Most eukaryotic organisms except for mammals, including fungi, insects, and plants, which have no antibody-mediated immune system, sense long exogenous dsRNAs as viruses and then activate the RNAi (PTGS) pathway for defence against virus infection ${ }^{6-8}$. In contrast, mammals sense long exogenous dsRNAs as virus infections and respond by activating the immune system, which has specifically developed innate and adaptive immune components for defence against pathogens ${ }^{9,10}$. Therefore, dsRNAs longer than 30 nucleotides (nt) are generally used to induce RNAi in fungi, insects and plants ${ }^{1,2,11}$, but in mammals, they often activate the innate immune system including the interferon pathway ${ }^{9,10}$. Shorter dsRNAs (siRNA duplexes) of 20 to 22 nt in length are typically used in RNAi experiments, as they prevent activation of the immune response in mammalian cells ${ }^{12}$. Furthermore, Kim et al. demonstrated that dsRNAs of 25 to $30 \mathrm{nt}$ in length induce RNAi as much as 100-fold more potently than 21-nt dsRNAs (siRNA duplexes), and this greater potency depends on processing of the 25- to 30-nt dsRNAs by Dicer ${ }^{13}$. They termed these dsRNAs "Dicer-substrate" siRNAs (dsiRNAs).

Although direct transfer of dsiRNAs or siRNAs into cultured cells is a standard protocol in RNAi experiments in mammals ${ }^{12,13}$, the direct transfer of dsRNAs into intact plant cells is difficult because of the cell wall. Therefore, because transgenic plants have been easily created since the 1980's, researchers have studied RNAi in plants by creating transgenic plants that express stem-loop (fold-back) single-stranded RNAs (ssRNAs) containing a long dsRNA region of 100 to $1,000 \mathrm{nt}^{14}$.

In plants, many important genes involved in RNAi have been discovered and characterized in genetic studies using mutants of the transgenic RNAi-expressing model plant, Arabidopsis thaliana ${ }^{15,16}$. However, characterizing the enzymatic properties of proteins that constitute the RNAi machinery in plant cells is difficult, because plant cells usually have a large central vacuole containing a variety of proteinases and nucleases. The biochemical

${ }^{1}$ Department of Applied Biological Sciences, Tokyo University of Agriculture and Technology, 3-5-8 Saiwaicho, Fuchu, Tokyo, 183-8509, Japan. ${ }^{2}$ Institute of Global Innovation Research, Tokyo University of Agriculture and Technology, 3-5-8 Saiwaicho, Fuchu, Tokyo, 183-8509, Japan. Sayaka Kakiyama and Midori Tabara contributed equally. Correspondence and requests for materials should be addressed toT.F. (email: fuku@cc.tuat.ac.jp) 


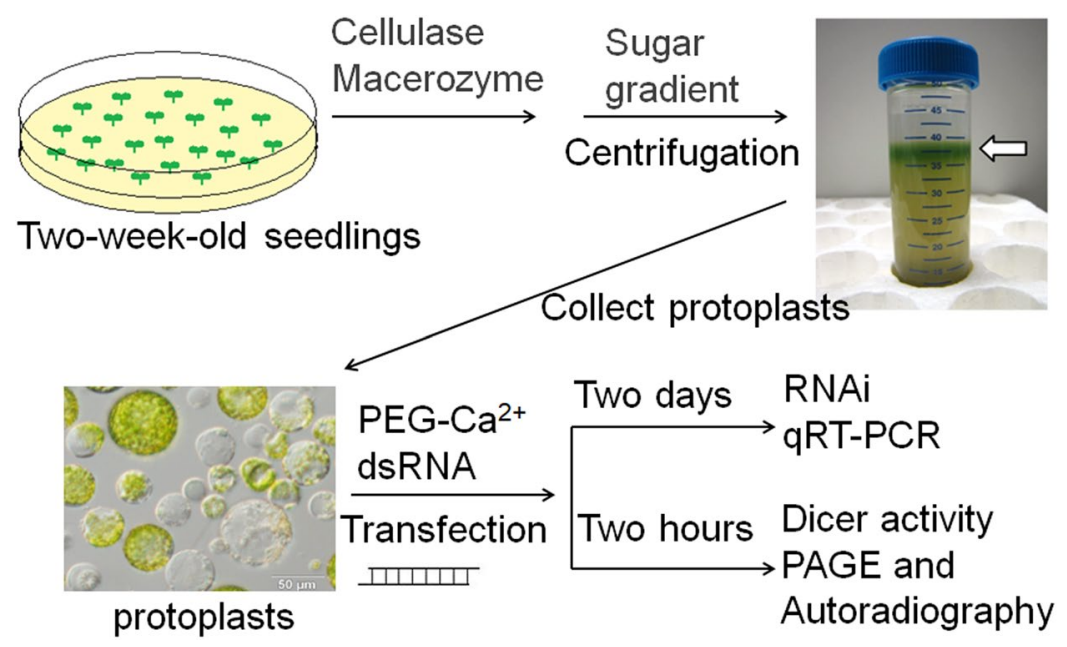

Figure 1. Schematic representation of the experimental procedure to examine the efficacies of various dsRNAs for inducing RNAi. Various dsRNAs were directly transferred into protoplasts prepared from 2-week-old Arabidopsis seedlings, and then their efficacies for inducing RNAi were analysed using qRT-PCR. DCL3 and DCL4 activities were analysed by denaturing PAGE and autoradiography. For details see Methods ${ }^{28,29}$.

properties of two core proteins (Dicer and Argonaute) in the RNAi machinery have been extensively studied in humans, the fruit fly Drosophila melanogaster, and the nematode Caenorhabditis elegans ${ }^{17-19}$. Therefore, even though some important genes encoding RNAi machinery components were first discovered in the model plant A. thaliana via genetic studies ${ }^{15,16}$, findings from biochemical studies related to the molecular mechanism of plant RNAi are limited.

We established a simple biochemical method to simultaneously monitor the dsRNA-cleaving activities of Dicer-like 3 (DCL3) and 4 (DCL4) in cell-free extracts of Arabidopsis seedlings ${ }^{20-23}$. We demonstrated that the enzymatic properties of DCL3 and DCL4 differ markedly in vitro and that they preferentially cleave short $(<50$ $\mathrm{nt})$ and long (>50 nt) dsRNAs, respectively ${ }^{20,21}$. The dicing activities of DCL3 and DCL4 are post-translationally regulated by inorganic phosphate and the redox state ${ }^{22}$. Our results from in vitro biochemical studies are consistent with reports indicating that RNA polymerase IV (Pol IV)-dependent RNAs, which are the precursors of 24-nt siRNAs produced by DCL3, are short ssRNAs of 30 to $40 \mathrm{nt}$ in length in vivo ${ }^{24,25}$. Our previous biochemical results are also consistent with the in vivo function of DCL4, in which the enzyme primarily cleaves long viral dsRNAs into 21-nt viral-derived siRNAs (vsiRNAs) in most virus-infected plant cells ${ }^{26,27}$.

It is difficult to evaluate the efficacies of various-sized dsRNAs in terms of inducing RNAi using transgenic plants expressing stem-loop ssRNAs containing a long dsRNA region ${ }^{14}$. Consequently, in this study, we evaluated the efficacies of various dsRNAs for inducing RNAi by direct transfer of dsRNAs into protoplasts prepared from Arabidopsis seedlings. Using this protoplast RNAi system, we compared the efficacies of various-sized dsRNAs for inducing RNAi and assessed the dsRNA-cleaving activities of DCL3 and DCL4 for long and short dsRNAs as substrates in Arabidopsis protoplasts. We demonstrated that DCL4 preferentially cleaves long dsRNAs ( $>100 \mathrm{nt})$ in protoplasts. Moreover, these long DCL4-substrate dsRNAs induce RNAi as much as 400-fold more potently than 21- or 37-nt dsRNAs. Therefore, the cleavage of long dsRNAs by DCL4 is essential for the efficient induction of RNAi in plant cells.

\section{Results}

DCL4 preferentially cleaves long dsRNAs into 21-nt RNAs in protoplasts. We established a simple biochemical method to simultaneously monitor the dsRNA-cleaving activities of DCL3 and DCL4 in cell-free extracts of Arabidopsis seedlings and demonstrated their enzymatic properties in vitro ${ }^{20-23}$. To monitor and characterize the dsRNA-cleaving activities of these Dicer enzymes in plant cells, we prepared protoplasts from 2-weekold seedlings of $A$. thaliana, transfected protoplasts with various ${ }^{32} \mathrm{P}$-labeled dsRNAs as substrates, extracted total RNAs, and then analysed cleaved RNA products using denaturing polyacrylamide gel electrophoresis (PAGE) and autoradiography (Figs 1 and 2). In protoplasts transfected with 130- and 500-nt dsRNAs, ${ }^{32}$ P-labeled 21-nt RNAs were detected, but no small RNA products ranging from 21 to $24 \mathrm{nt}$ were detected in protoplasts transfected with 37-nt dsRNAs (Fig. 2A). Based on size, the ${ }^{32}$ P-labeled 21-nt RNAs were expected to be DCL4 cleavage products. As no cleaved products were detected in protoplasts prepared from $d c l 4$ mutant seedlings (Fig. 2B), they were confirmed as products of DCL4 cleavage. These results indicate that DCL4 preferentially cleaves long dsRNAs (>100 nt) into 21-nt RNAs in protoplasts (plant cells), confirming our previous finding demonstrating that DCL4 preferentially cleaves long dsRNAs into 21 -nt dsRNAs in vitro ${ }^{21}$. However, we could not detect activity of DCL3 (which produces 24-nt siRNAs) in protoplasts (Fig. 2) even though the dsRNA-cleavage of short 37-nt substrate dsRNAs by DCL3 was easily detected in an in vitro biochemical assay ${ }^{21}$. Although the weak dicing activity of DCL4 to 37-nt dsRNAs was also detected in an in vitro assay ${ }^{21}$, we could not detect 21-nt RNA products from 37-nt dsRNAs by DCL4 (Fig. 2A). The results indicated that the sensitivity to detect the dicing activity in protoplasts is lower than that in an in vitro biochemical assay ${ }^{21}$. 


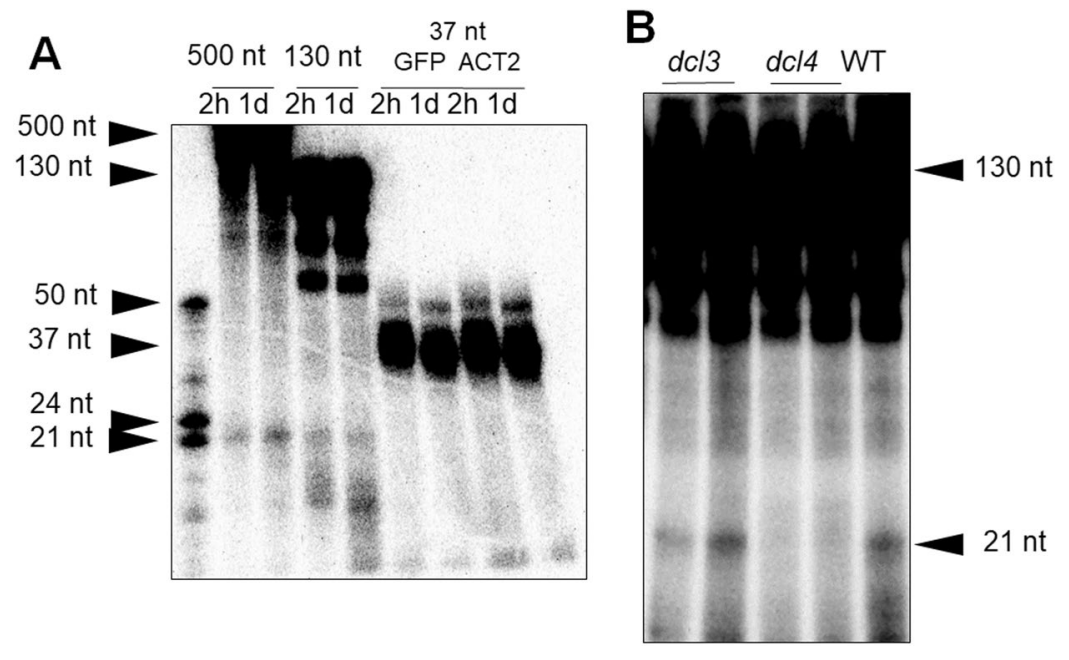

Figure 2. The dsRNA-cleaving activity of DCL4 in Arabidopsis protoplasts. Protoplasts prepared from 2-weekold Arabidopsis seedlings were transfected with ${ }^{32}$ P-labeled dsRNAs of $37 \mathrm{nt}(\mathbf{A}), 130 \mathrm{nt}(\mathbf{A}, \mathbf{B})$, or $500 \mathrm{nt}(\mathbf{A})$. Total RNAs were extracted from protoplasts 2 hours $(\mathbf{A})$ or 1 day $(\mathbf{A}, \mathbf{B})$ after transfection, and electrophoresed by denaturing 15\% PAGE. Cleaved products were detected by autoradiography. Protoplasts were isolated from WT (A,B), dcl3 (B), or dcl4 (B) seedlings, and the 37-nt dsRNAs (A) were synthesized from the ACT2 and GFP genes and dsRNAs of $130 \mathrm{nt}$ and $500 \mathrm{nt}$ were synthesized from the GFP gene. Two biological replicates are shown for lanes $d c l 3$ and $d c l 4$ in (B).

Long dsRNAs of approximately $130 \mathrm{nt}$ induce RNAi much more effectively than short dsRNAs of $37 \mathrm{nt}$. As we demonstrated that DCL3 preferentially cleaves short dsRNAs of $<50 \mathrm{nt}$ into 24 -nt dsRNAs and DCL4 preferentially cleaves long dsRNAs of $>50 \mathrm{nt}$ into 21 -nt dsRNAs in vitro ${ }^{21}$, we examined the efficacies of various-sized dsRNAs for inducing RNAi by directly transferring dsRNAs into protoplasts. We synthesized 37-nt and approximately 130-nt dsRNAs from Green Fluorescent Protein (GFP), Arabidopsis ACTIN2 (ACT2), and ELONGATION FACTOR-1 $\alpha(E F-1 \alpha)$ complementary DNAs (cDNAs) in vitro and used them to transfect protoplasts prepared from wild-type (WT) and GFP-over-expressing Arabidopsis seedlings (Figs 3-5). Two days after transfection, the efficacies of the dsRNAs for inducing RNAi were evaluated microscopically determining the fluorescence intensity of protoplasts (Fig. 3A) using ImageJ software (Fig. 3B). In addition, relative mRNA accumulation was assessed using quantitative real-time PCR (qRT-PCR) (Figs 3C-5).

In GFP-overexpressing protoplasts transfected with GFP dsRNAs, the green fluorescence of the protoplasts (Fig. 3A,B) and the relative abundance of GFP transcripts (Fig. 3C) were reduced, but only in protoplasts transfected with the 130-nt GFP dsRNA. In WT protoplasts transfected with EF-1 $\alpha$ and ACT2 dsRNAs (Figs 4A and 5A), long dsRNAs of approximately $130 \mathrm{nt}$ induced RNAi much more effectively than short dsRNAs of 21 to $37 \mathrm{nt}$. In all three experiments in which different dsRNAs were transfected (Figs 3-5), the abundance of target transcripts in protoplasts transfected with long dsRNAs relative to the abundance in mock-transfected protoplasts was lowest (about 10 to 15\%). Knockdown levels of target mRNAs (10 to 15\%) in protoplasts transfected with $3 \mu$ g of long dsRNAs were high relative to previous reports involving this protoplast RNAi system ${ }^{28,29}$. However, there was no or little decrease in the relative abundance of target transcripts in protoplasts transfected with short dsRNAs of 21,24 or 37 nt relative to protoplasts transfected with long dsRNAs.

Although these three genes (particularly GFP, which is driven by the cauliflower mosaic virus 35 S promoter) are highly and constitutively expressed ${ }^{30,31}$, direct transfer of long dsRNAs into protoplasts efficiently reduced the expression of these genes to approximately $10 \%$ of the control (Figs 3-5).

Dose dependence of transfected dsRNAs for inducing RNAi. As the transfection of protoplasts with $3 \mu \mathrm{g}$ of long dsRNAs seemed sufficient to induce efficient knockdown of target gene expression (10 to $15 \%$ ), the dose dependence of transfected dsRNAs for inducing RNAi was examined by directly transfecting WT protoplasts with varying amounts of EF-1 $\alpha$ (Fig. $4 \mathrm{~B}$ ) and ACT2 (Fig. 5B) dsRNAs.

In both transfection experiments (Figs $4 \mathrm{~B}$ and $5 \mathrm{~B}$ ), approximately $300 \mathrm{ng}$ of 130 -nt dsRNAs were sufficient to reduce transcript levels to $<20 \%$, and transfections of longer dsRNAs appeared to saturate the knock-down of gene expression. From the results shown in Fig. 4B, approximately $4.5 \mathrm{ng}$ of $139-\mathrm{nt} E F-1 \alpha$ dsRNA was estimated to be sufficient to induce a $50 \%$ reduction in $E F-1 \alpha$ transcript level, but approximately $1.8 \mu \mathrm{g}$ of 37 -nt $E F-1 \alpha$ dsRNA would be needed to reduce the transcript level by $50 \%$. The efficacy of the 139 -nt dsRNA for inducing RNAi was thus 400-fold greater than that of the 37-nt dsRNA (Fig. 4B). The same calculation was carried out using the data shown in Fig. 5B (ACT2) and revealed that the efficacy of the 127-nt ACT2 dsRNA for inducing RNAi was 60-fold greater than that of the 37-nt ACT2 dsRNA. Therefore, regardless of whether target genes are exogenous (GFP) or endogenous (EF-1 $\alpha$ and ACT2), the direct transfer of long dsRNAs of approximately $130 \mathrm{nt}$ efficiently induces RNAi in protoplasts. 
A

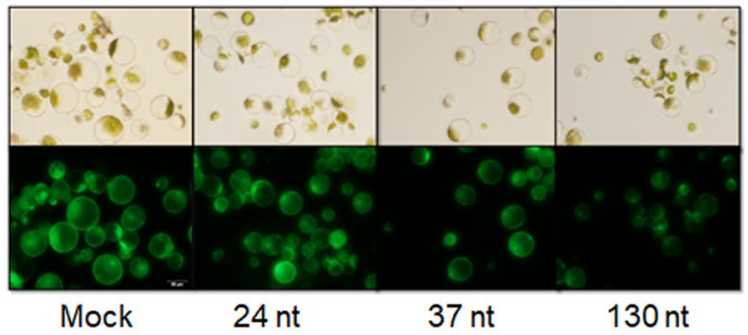

C

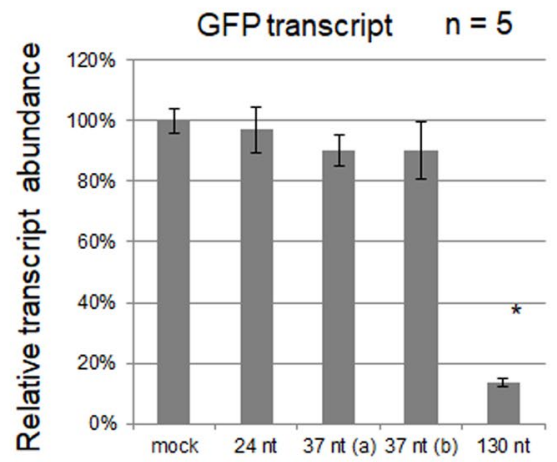

B

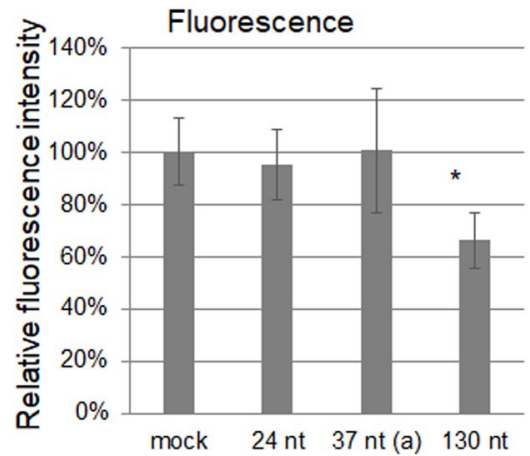

${ }^{*} p=0.05$

$$
{ }^{*} \mathrm{p}<0.005
$$

Figure 3. RNAi (gene silencing) for the exogenous GFP gene was successfully induced by direct transfer of long dsRNAs of about $130 \mathrm{nt}$ into protoplasts. Approximately $3 \mu \mathrm{g}$ of dsRNA was transfected into $0.1 \mathrm{~mL}$ of protoplasts $\left(10^{6}\right.$ to $10^{7}$ cells $/ \mathrm{mL}$ ) prepared from 2 -week-old Arabidopsis seedlings over-expressing the GFP gene. The efficacies of various-sized dsRNAs for inducing RNAi were evaluated by $(\mathbf{A}, \mathbf{B})$ observing green fluorescence by microscopy and (C) analysing the relative abundance of GFP transcripts by qRT-PCR. (A) Bright-field (upper panels) and fluorescence (lower panels) micrographs of protoplasts transfected without (mock) or with $3 \mu \mathrm{g}$ of various-sized dsRNAs against GFP (RNAi) were examined, and the fluorescence was quantified. Fluorescence micrographs were photographed 2 days after transfection. All images were captured for the same exposure period. (B) Fluorescence intensities of dsRNA-transfected protoplasts relative to that of mock-transfected protoplasts $(100 \%)$ were calculated using Image J software. Error bars indicate the standard errors of three replicates, and the p-value was determined using the Student's $t$-test. (C) The relative abundance of GFP transcripts in dsRNA-transfected protoplasts compared to mock-transfected protoplasts (100\%) was determined by qRT-PCR. Protoplasts in $100 \mu \mathrm{L}\left(10^{6}\right.$ to $10^{7}$ cells $\left./ \mathrm{mL}\right)$ were transfected without (mock) or with $3 \mu \mathrm{g}$ of various-sized dsRNAs against the exogenous GFP gene (RNAi). Two 37-nt dsRNAs ( $a$ and $b$ ) with a different nucleotide at each end were used. Total RNAs were extracted from protoplasts 2 days after transfection. The efficacies of dsRNAs for inducing RNAi were analysed by qRT-PCR for the relative abundance of GFP transcripts normalized to ACT2 transcripts (house-keeping gene). Error bars indicate the standard errors of five biological replicates, and p-values were determined using the Student's $t$-test. Asterisks $(*)$ in $(\mathbf{B}, \mathbf{C})$ indicate significant differences between mock and 130-nt dsRNA transfections.

These results are consistent with our previous results demonstrating that DCL4 cleaves long dsRNAs $>50$ nt efficiently and 37-nt dsRNAs slightly in vitro ${ }^{21}$. They are also consistent with the results shown in Fig. 2 that cleavage products of 21-nt by DCL4 were detected from 130-nt dsRNAs but not from 37-nt dsRNAs in protoplasts. As DCL4 is essential for RNAi in plants ${ }^{32,33}$ and long dsRNAs are preferred substrates for DCL4 in plant cells (protoplasts), they are able to induce RNAi more efficiently than short dsRNAs. Because short dsRNAs of 37 nt did induce RNAi ineffectively (Figs 4 and 5), 21-nt siRNAs must be produced from 37-nt dsRNAs by DCL4 in protoplasts but they were not detected (Fig. 2).

Cleavage of long dsRNAs by DCL4 is essential for efficient induction of RNAi in protoplasts. Short dsRNAs (siRNA duplexes) of 21 to $24 \mathrm{nt}$ are produced from long dsRNAs by three Dicers (DCL2, DCL3 and DCL4) in plants, so the direct transfer of 21-nt dsRNAs into protoplasts must induce RNAi without Dicer activity. As 21-nt siRNAs produced by DCL4 generally induce RNAi (mRNA cleavage) but 24-nt siRNAs produced by DCL3 induce RNA-directed DNA methylation (RdDM), we transfected protoplasts with 21-nt dsRNAs (RNAi) and 24-nt dsRNAs (RdDM).

The 21-nt dsRNA (b) induced RNAi more efficiently than 21-nt dsRNA (a) and 24-nt dsRNA (Fig. 5). It is therefore reasonable to conclude that the 21-nt dsRNA (b) is more efficient than the 24-nt dsRNA, because 21-nt siRNAs induce RNAi and 24-nt siRNAs induce RdDM. Interestingly, the efficacy of two different 21-nt dsRNAs 

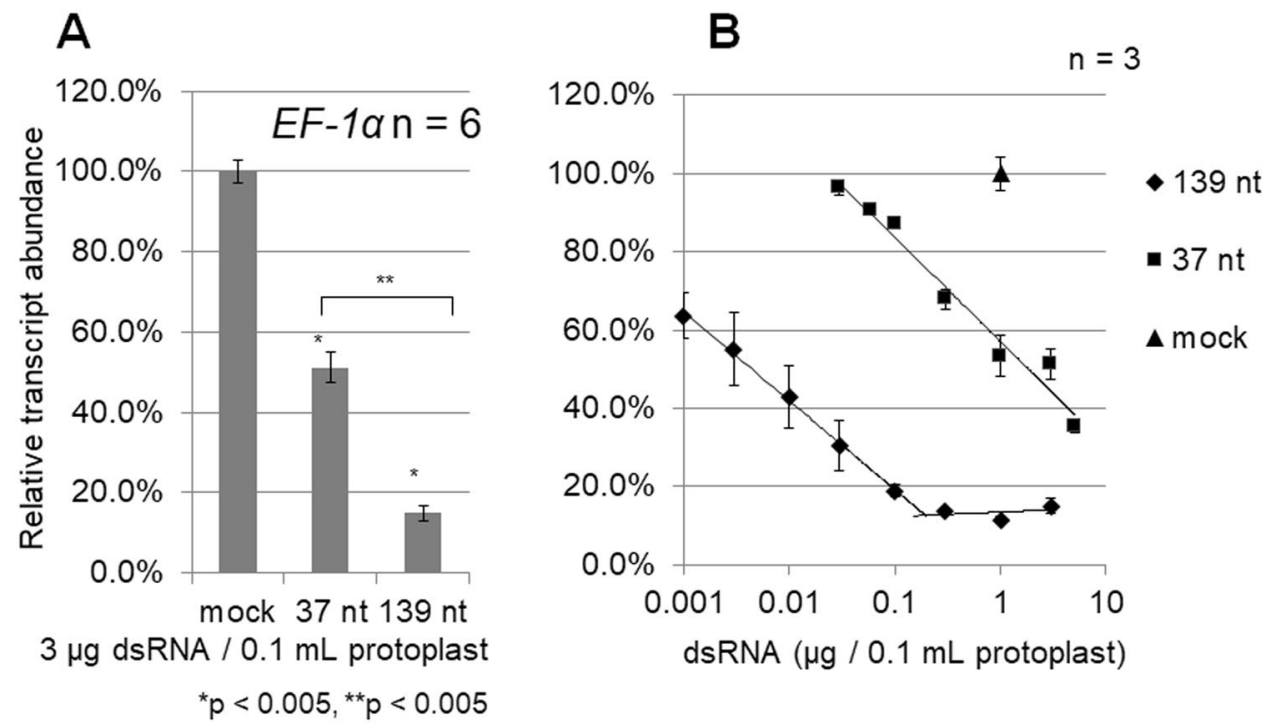

Figure 4. A long dsRNA of $139 \mathrm{nt}$ is much more effective (approximately 400 -fold) at inducing RNAi of the endogenous EF-1 $\alpha$ gene than a short dsRNA of $37 \mathrm{nt}$. (A) Direct transfer of dsRNAs successfully induced RNAi of the endogenous EF- $1 \alpha$ gene, which is a typical house-keeping gene ${ }^{31}$. Protoplasts in $100 \mu \mathrm{L}\left(10^{6}\right.$ to $10^{7}$ cells $/ \mathrm{mL}$ ) were transfected without (mock) or with $3 \mu \mathrm{g}$ of short (37 nt) or long (139 nt) dsRNA against the $E F-1 \alpha$ gene (RNAi). Protoplasts were collected 2 days after transfection. The abundance of $E F-1 \alpha$ transcripts in dsRNA-transfected protoplasts relative to that in mock-transfected protoplasts (100\%) was determined by qRT-PCR and normalized to the abundance of ACT2 transcripts. Error bars indicate the standard errors of six biological replicates, and p-values were determined using the Student's $t$-test. Single asterisks $(*)$ indicate significant differences between mock- and dsRNA-transfected (37 nt and $139 \mathrm{nt})$ protoplasts, and double asterisk $(* *)$ indicates a significant difference between $37 \mathrm{nt}$ and $139 \mathrm{nt}$ dsRNA-transfected protoplasts. (B) Dose dependence of dsRNAs for inducing RNAi of the endogenous $E F-1 \alpha$ gene in protoplasts was examined by transfecting protoplasts with varying amounts $(0.001$ to $5 \mu \mathrm{g})$ of 37-nt or 139-nt dsRNAs corresponding to the $E F-1 \alpha$ gene. The relative abundance of $E F-1 \alpha$ transcripts in protoplasts was determined by qRT-PCR and normalized to the abundance of ACT2 transcripts. The amount of 139-nt dsRNA needed to reduce the level of $E F-1 \alpha$ transcripts (RNAi) by $50 \%$ was estimated approximately $0.0045 \mu \mathrm{g}$, whereas the amount of 37 -nt dsRNA needed to reduce the level of $E F-1 \alpha$ transcripts (RNAi) by $50 \%$ was estimated at $1.84 \mu \mathrm{g}$. Error bars indicate the standard error of three biological replicates.

for inducing RNAi differed depending on their sequences at both ends of each dsRNA (compare $21 \mathrm{nt}$ [a] with 21 nt $[\mathrm{b}]$ in Fig. 5C). If the guide-strand selection rule for AGO proteins (demonstrated primarily in a biochemical study using fruit fly extracts ${ }^{34}$ ) is applicable to plant AGO proteins ${ }^{35}$, the 21-nt dsRNA (a) sense strand could be preferentially loaded onto AGO1 as the guide strand (Fig. 5C). However, AGO1 with the 21-nt dsRNA (a) sense strand cannot slice ACT2 mRNA. The 21-nt dsRNA (b) antisense strand can be loaded onto AGO1, and this complex can slice ACT2 mRNA. Furthermore, as the nucleotide at the $5^{\prime}$-ends of the antisense strands of 21 -nt dsRNA (a) and (b) are cytidine and uridine, respectively, they can be loaded onto AGO5 and AGO1, respectively ${ }^{36,37}$. Experiments examining this interesting relationship between 21-nt dsRNA nucleotide sequences and their efficacy for inducing RNAi are now in progress. Even though the $21 \mathrm{nt}$ dsRNA (b) was more efficient than the 21-nt dsRNA (a), the long dsRNA of approximately 130 nt induced RNAi much more efficiently than the 21-nt dsRNA (b) (Fig. 5B).

The 127-nt ACT2 dsRNA induced RNAi about 100-fold more efficiently than the 21-nt (b) dsRNA (Fig. 5B), suggesting that cleavage of long dsRNAs into 21-nt dsRNAs (siRNA duplexes) by DCL4 is essential for efficient induction of RNAi in plants. RNAi enhancement via the cleavage of dsRNA substrates by Dicer enzymes has been reported in mammalian cells ${ }^{13}$, in which it was demonstrated that dsRNAs of 25 to 30 nt induce RNAi up to 100 -fold more potently than 21-nt dsRNAs in human cells. In plant protoplasts (Fig. 5A,B), however, 37-nt dsRNAs were as efficient as the 21-nt dsRNA (21 nt [b]). The reason for the ineffective induction of RNAi in plant cells by 37-nt dsRNAs (Figs 3-5) may be the ineffective cleavage of 37-nt dsRNAs by DCL4 in protoplasts (Fig. 2) and in vitro ${ }^{21}$.

dsRNA-induced RNAi in protoplasts depends primarily on DCL4. To confirm the involvement of DCL4 in inducing RNAi in protoplasts, we transfected protoplasts prepared from $\mathrm{dcl}$-mutant seedlings. In experiments in which $0.3 \mu \mathrm{g}$ of 139 -nt $E F-1 \alpha$ dsRNA was transfected into protoplasts prepared from seedlings of WT, $d c l 2$ single, $d c l 4$ single, and $d c l 2 d c l 4$ double mutants, less RNAi was induced in the $d c l 4$ and $d c l 2 d c l 4$ protoplasts, with the $E F-1 \alpha$ transcript abundance reduced to only approximately $80 \%$ of the control (Fig. 6A), indicating that DCL4 is primarily involved in inducing RNAi in protoplasts, as shown in previous genetic studies ${ }^{32,33}$. The efficiency of RNAi induction in $d c l 2$ protoplasts (approximately $40 \%$ ) was intermediate between WT (approximately 


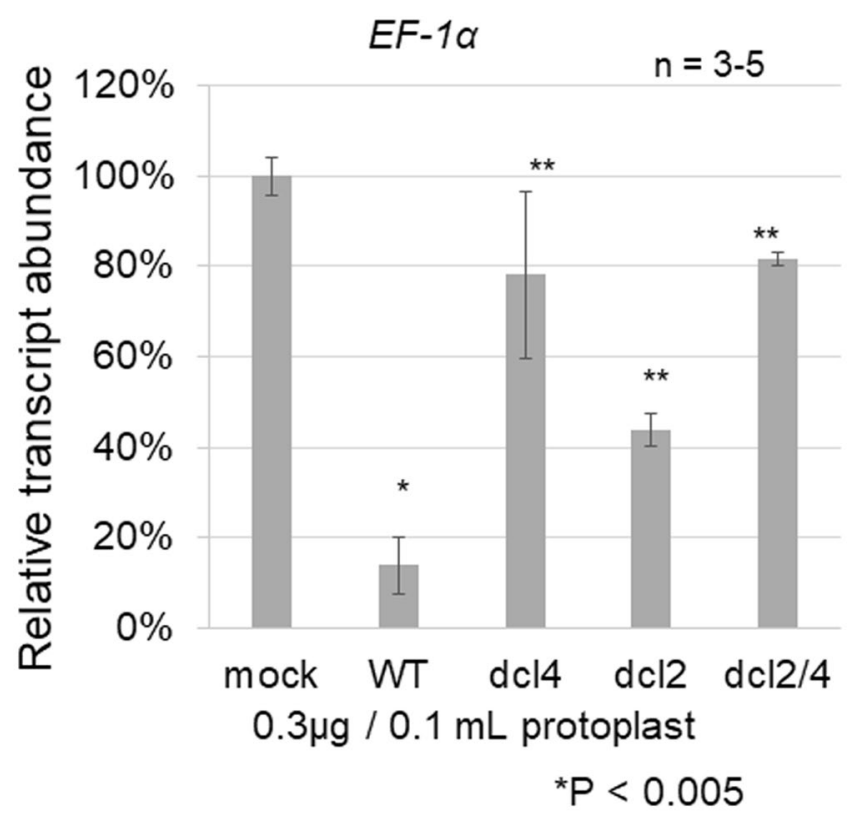

Figure 6. Efficiency of RNAi in $d c l$ mutants. A $0.1-\mathrm{mL}$ volume of protoplasts $\left(10^{6}\right.$ to $10^{7}$ cells $\left./ \mathrm{mL}\right)$ prepared from Arabidopsis WT or $d c l$ mutant seedlings were transfected with $0.3 \mu \mathrm{g}$ of 139 -nt $E F-1 \alpha$ dsRNA. Protoplasts were collected 2 days after transfection, and then the abundance of $E F-1 \alpha$ transcripts in transfected protoplasts relative to that in mock-transfected protoplasts (100\%) was determined by qRT-PCR and normalized to the level of ACT2 transcripts. Error bars indicate the standard error of three to five biological replicates, and p-values were determined using the Student's $t$-test. Single asterisk $\left.*^{*}\right)$ indicates significant difference between mock- and 139-nt dsRNA-transfected WT protoplasts, and double asterisks $(* *)$ indicate significant differences between WT and the three mutant protoplasts.

The dsRNA-cleaving activity of DCL3, which cleaves 37-nt dsRNA into 24-nt RNA, can be readily detected in crude extracts prepared from 2 -week-old Arabidopsis seedlings using an in vitro biochemical assay ${ }^{21,22}$. However, no products of cleavage of 37-nt dsRNAs by DCL3 were detected in protoplasts in the present study, although products of cleavage of 130-nt dsRNAs by DCL4 were detected (Fig. 2). The subcellular localizations of DCL3 and DCL4 may explain this discrepancy, as DCL3 is localized and functions in the nucleus ${ }^{40,41}$, whereas DCL4 is localized primarily in the cytoplasm ${ }^{42,43}$. Transfection of inducer dsRNAs into the cytoplasm in the protoplast, as occurs in the RNAi system we used, could render it impossible to detect DCL3 activity under the conditions used in our experiments.

DCL2 and DCL4 cleave viral and transgene-derived dsRNAs into 22- and 21-nt siRNAs, respectively, and they function redundantly and hierarchically in virus resistance and RNAi ${ }^{30,31,36,37}$. DCL4 functions as a primary Dicer (DCL) for virus resistance and RNAi, whereas DCL2 serves as a functional backup in the absence of DCL4. The results shown in Fig. 6 indicate that DCL2 and DCL4 also function redundantly and hierarchically in RNAi in plant protoplasts.

Long dsRNAs of $130 \mathrm{nt}$ exhibited greater efficiency for inducing RNAi than 21-nt dsRNAs, which are not necessary for DCL4 activity to induce RNAi. Although a similar phenomenon has been reported in mammalian cells ${ }^{13}$, this is the first such report in plants. Kim et al. noted that the enhanced induction of RNAi by dsiRNAs in mammalian cells results from the close link between Dicer cleavage of dsRNAs into siRNAs and loading of cleaved siRNAs onto AGO proteins ${ }^{13}$. Our results suggest that a similar link between Dicer cleavage and AGO loading exists in plant cells and that DCL4-cleaved siRNAs (i.e., not free siRNAs) may also efficiently load onto AGOs to induce RNAi in plant cells.

Short dsiRNAs of 25 to $30 \mathrm{nt}$ are typically used for RNAi experiments in mammalian cells because they do not activate the innate immune response, including the interferon pathway, ${ }^{9,10,13}$. In contrast, in this study, we demonstrated that short dsRNAs of $37 \mathrm{nt}$ are not potent inducer of RNAi in plant cells because they are not cleaved by DCL4, whereas long dsRNAs of $130 \mathrm{nt}$ potently induce RNAi because they are preferentially cleaved by DCL4. Therefore, the results of RNAi experiments in plant protoplasts reported here are consistent with our previous results regarding the substrate specificities of DCL3 and DCL4 in vitro ${ }^{21}$. Moreover, these results suggest that DCL3 and DCL4 function according to their substrate specificities in vivo. In mammals, long dsRNAs $>30$ nt activate the innate immune system ${ }^{9,10}$, whereas short Dicer-substrate dsRNAs of 25 to 30 nt efficiently induce the RNAi pathway without activating an immune response ${ }^{12,13}$. Although long DCL4-substrate dsRNAs efficiently induce RNAi in plants, short DCL3-substrate dsRNAs of 30 to 37 nt might induce RdDM but not RNAi ${ }^{21,24,25}$. Experiments examining the induction of RdDM by the direct transfer of short dsRNAs into protoplasts are now in progress. 


\begin{abstract}
Methods
Plant material and growth conditions. WT and $d c l$ mutant $A$. thaliana (Columbia ecotype) seeds were sterilized in $70 \%$ ethanol, and $6 \%$ sodium hypochlorite with $0.1 \%$ Tween 20 . Seeds were sown on Murashige and Skoog (MS) medium supplemented with $1 \%$ sucrose and $0.7 \%$ agar and then kept for $48 \mathrm{~h}$ at $4{ }^{\circ} \mathrm{C}$ in the dark for stratification. Seeds were germinated and seedlings were grown in a 16-h-light photoperiod at $22^{\circ} \mathrm{C}$.

Seeds of $d c l 2-1$ (CS16389) and dcl3-1 (SALK_005512) were kindly provided by the Arabidopsis Biological Resource Center (ABRC, USA), and seeds of $d c l 4-2$ (GABI 160G05) were kindly provided by the Genomanalyse im Biologischen System Pflanze (Germany). Seeds of a transgenic Arabidopsis variety that over expresses GFP were kindly provided by Dr. Yasuo Niwa (University of Shizuoka, Japan) ${ }^{30}$.
\end{abstract}

Isolation of protoplasts from Arabidopsis seedlings. Protoplasts were isolated from Arabidopsis seedlings using a modified method of Zhai et al. ${ }^{28}$. Approximately $2 \mathrm{~g}$ of 2 -week-old Arabidopsis seedlings were sliced with a razor blade into stripes of approximately $1 \mathrm{~mm}$ in $15 \mathrm{~mL}$ of filter-sterilized TVL solution $(0.3 \mathrm{M}$ sorbitol and $50 \mathrm{mM} \mathrm{CaCl}_{2}$ ). Next, $20 \mathrm{~mL}$ of enzyme solution (0.5 M sucrose, $20 \mathrm{mM} \mathrm{MES-KOH} \mathrm{(pH} \mathrm{5.7),} 20 \mathrm{mM} \mathrm{CaCl}_{2}$, $40 \mathrm{mM} \mathrm{KCl}, 1 \%$ cellulase [Onozuka R-10], and 1\% Macerozyme [R10]) was added to the sliced seedlings, and the mixture was agitated at $35 \mathrm{rpm}$ in the dark at $22^{\circ} \mathrm{C}$. After 16 to $18 \mathrm{~h}$, the released protoplasts were sieved through a nylon mesh $(80 \mu \mathrm{m})$ and transferred into $50-\mathrm{mL}$ centrifuge tubes. Protoplasts and plant debris retained on the nylon mesh were gently sieved-through one more time by washing the mesh with $5 \mathrm{~mL}$ of W5 solution $(0.1 \%$ glucose, $0.08 \% \mathrm{KCl}, 0.9 \% \mathrm{NaCl}, 1.84 \% \mathrm{CaCl}_{2}$, and $2 \mathrm{mM}$ MES-KOH [pH 5.7]). Sieved-through protoplasts were combined in a $50-\mathrm{mL}$ centrifuge tube, overlaid with $10 \mathrm{~mL}$ of W5 solution, and centrifuged for $20 \mathrm{~min}$ at $100 \mathrm{~g}$ at $22^{\circ} \mathrm{C}$. Approximately $10 \mathrm{~mL}$ of the protoplast fraction was collected from the interface of the enzyme and W5 solutions (Fig. 1). Protoplasts were washed twice with $15 \mathrm{~mL}$ of W5 solution and suspended in 1 to $2 \mathrm{~mL}$ of W5 solution. The concentration of protoplasts was measured by cell counting using a hemocytometer.

Preparation of dsRNAs. DNA templates for short dsRNAs $<37 \mathrm{nt}$ were designed from cDNA sequences

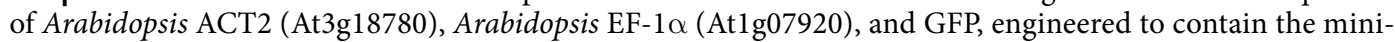
mal T7-RNA polymerase promoter sequence (TAATACGACTCACTATAGGG) at both the $5^{\prime}$ and $3^{\prime}$ ends, and then synthesized by Sigma-Aldrich (Japan). DNA templates for long dsRNAs were synthesized by PCR using KOD-plus DNA polymerase (Toyobo, Japan) from plasmids containing an Arabidopsis cDNA. Nucleotide sequences of DNA templates for short dsRNA syntheses and primers used to amplify DNAs of target genes for long dsRNA syntheses are listed in Table S1. dsRNAs were synthesized using the in vitro T7 Transcription kit for siRNA synthesis (Takara, Japan) according to the manufacturer's recommendations (http://catalog.takarabio. co.jp/product/basic_info.php?unitid=U100004288). A 2-nt 3' overhang of dsRNAs was made using RNase T1, and DNA templates were removed with RNase-free DNase I (Takara). dsRNAs were extracted by phenol/chloroform/isoamyl alcohol (25:24:1), precipitated with an equal volume of $5 \mathrm{M}$ ammonium acetate and 4 volumes of ethanol, and reconstituted with ST buffer $(10 \mathrm{mM}$ Tris- $\mathrm{HCl}[\mathrm{pH} 7.5]$ and $100 \mathrm{mM} \mathrm{NaCl})$. The quality and quantity of synthesized dsRNAs were determined by agarose gel electrophoresis (see Supplementary Fig. S2) and UV spectrophotometry (NanoDrop Lite Microlitre spectrophotometer, Labtech International Ltd., UK).

Transfection of protoplasts with dsRNAs. Protoplasts were transfected using a modified method of Jung et al. ${ }^{29}$. Briefly, protoplasts in W5 solution were incubated on ice for $30 \mathrm{~min}$, after which the W5 solution was discarded, and protoplasts were resuspended in MMG solution (4 mM MES-KOH [pH5.7], 0.4 M mannitol, and $15 \mathrm{mM} \mathrm{MgCl}_{2}$ ) to a final concentration of $10^{6}$ to $10^{7}$ protoplasts $/ \mathrm{mL}$. Aliquots of protoplasts $(100 \mu \mathrm{L})$ were transferred into a $2-\mathrm{mL}$ round-bottom microcentrifuge tube and mixed gently with dsRNA ( 0.001 to $5 \mu \mathrm{g})$ and $110 \mu \mathrm{L}$ of PEG-calcium solution (40\% PEG-4000, 0.2 M mannitol, and $100 \mathrm{mM} \mathrm{CaCl}_{2}$ ). In negative control transfections, dsRNA was omitted, and an equivalent volume of deionized, sterile water was used for mock-transfection. Protoplasts were mixed with PEG-calcium solution by gently tapping the tube and incubated for $7 \mathrm{~min}$ at room temperature. Transfection was terminated by diluting the mixture with $600 \mu \mathrm{L}$ of W5 solution. Transfected protoplasts were collected by centrifugation for $2 \mathrm{~min}$ at $100 \mathrm{~g}$, resuspended in $1 \mathrm{~mL}$ of W5 solution, and kept in the dark for 2 days.

qRT-PCR. Transfected protoplasts $\left(10^{6}\right.$ to $10^{7}$ cells $\left./ \mathrm{mL}\right)$ were collected by centrifugation for 2 to $5 \mathrm{~min}$ at $100 \mathrm{~g}$. Total RNAs were extracted with $1 \mathrm{~mL}$ of TRIzol reagent following the manufacturer's protocol (Thermo Fisher Scientific, Japan). cDNAs were produced from total RNAs using a PrimeScript RT reagent kit with gDNA Eraser (Takara), and qRT-PCR was performed using a Thermal Cycler Dice Real Time System with a SYBR Premix Ex Taq II kit (Takara). Primers for qRT-PCR are listed in Table S2. The effectiveness of RNAi was evaluated by comparing the relative abundance of RNAi-target transcripts to transcripts of Arabidopsis house-keeping genes, such as $A C T 2$ and $E F-1 \alpha^{31}$.

Monitoring of Dicer (dsRNA-cleaving) activity in protoplasts. Methods for the preparation of $5^{\prime}{ }^{32} \mathrm{P}$-labelled short dsRNAs of $37 \mathrm{nt}$ and $\left[\alpha^{-32} \mathrm{P}\right]$-UTP-labeled long dsRNAs of approximately $130 \mathrm{nt}$ and 500 nt were described previously (Table S3) ${ }^{20,21}$. Nucleotide sequences of 37-nt dsRNAs are shown in Table S3. Protoplasts were transfected with ${ }^{32} \mathrm{P}$-labelled short and long dsRNAs, and then incubated for $2 \mathrm{~h}$ at room temperature. Total RNAs were extracted from protoplasts using phenol/chloroform/isoamyl alcohol (25:24:1), precipitated with ethanol, separated using 15\% denaturing PAGE with $8 \mathrm{M}$ urea, and detected by autoradiography (Typhoon FLA 7000 image analyser, GE Healthcare).

Fluorescence intensity quantification. GFP-expressing protoplasts were observed under light microscopy (IX71, Olympus, Tokyo, Japan) with a fluorescence excitation filter (470-495 nm) and emission filter (510$550 \mathrm{~nm}$ ). Fluorescence intensities of more than 20 protoplasts were quantified as gray values, and the area of each 
protoplast was calculated using ImageJ software (https://imagej.nih.gov/ij/). Relative fluorescence intensities were calculated as gray values normalized to the average protoplast area; three photographs containing more than 20 protoplasts were analysed.

\section{References}

1. Napoli, C., Lemieux, C. \& Jorgensen, R. Introduction of a chimeric chalcone synthase gene into petunia results in reversible cosuppression of homologous genes in trans. Plant Cell 2, 279-289 (1990).

2. Fire, A. et al. Potent and specific genetic interference by double-stranded RNA in Caenorhabditis elegans. Nature 391, 806-811 (1998).

3. Bernstein, E., Caudy, A. A., Hammond, S. M. \& Hannon, G. J. Role for a bidentate ribonuclease in the initiation step of RNA interference. Nature 409, 363-366 (2001).

4. Fagard, M., Boutet, S., Morel, J. B., Bellini, C. \& Vaucheret, H. AGO1, QDE-2, and RDE-1 are related proteins required for posttranscriptional gene silencing in plants, quelling in fungi, and RNA interference in animals. Proc. Natl. Acad. Sci. USA 97, 11650-11654 (2000).

5. Hammond, S. M., Boettcher, S., Caudy, A. A., Kobayashi, R. \& Hannon, G. J. Argonaute2, a link between genetic and biochemical analyses of RNAi. Science 293, 1146-1150 (2001).

6. Hamilton, A. J. \& Baulcombe, D. C. A species of small antisense RNA in posttranscriptional gene silencing in plants. Science 286, 950-952 (1999).

7. Baulcombe, D. RNA silencing in plants. Nature 431, 356-363 (2004).

8. Lindbo, J. A. \& Dougherty, W. G. Plant pathology and RNAi: a brief history. Annu. Rev. Phytopathol. 43, 191-204 (2005).

9. Manche, L., Green, S. R., Schmedt, C. \& Mathews, M. B. Interactions between double-stranded RNA regulators and the protein kinase DAI. Mol. Cell. Biol. 12, 5238-5248 (1992).

10. Stark, G. R., Kerr, I. M., Williams, B. R., Silverman, R. H. \& Schreiber, R. D. How cells respond to interferons. Annu. Rev. Biochem. 67, 227-264 (1998).

11. Romano, N. \& Macino, G. Quelling: transient inactivation of gene expression in Neurospora crassa by transformation with homologous sequences. Mol. Microbiol. 6, 3343-3353 (1992).

12. Elbashir, S. M. et al. Duplexes of 21-nucleotide RNAs mediate RNA interference in cultured mammalian cells. Nature 411, 494-498 (2001).

13. Kim, D. H. et al. Synthetic dsRNA Dicer substrates enhance RNAi potency and efficacy. Nat. Biotechnol. 23, 222-226 (2005).

14. Wesley, S. V. et al. Construct design for efficient, effective and high-throughput gene silencing in plants. Plant J. 27, 581-590 (2001).

15. Dalmay, T., Hamilton, A., Rudd, S., Angell, S. \& Baulcombe, D. C. An RNA-dependent RNA polymerase gene in Arabidopsis is required for posttranscriptional gene silencing mediated by a transgene but not by a virus. Cell 101, 543-553 (2000).

16. Mourrain, P. et al. Arabidopsis SGS2 and SGS3 genes are required for posttranscriptional gene silencing and natural virus resistance. Cell 101, 533-542 (2000).

17. Ketting, R. F. et al. Dicer functions in RNA interference and in synthesis of small RNA involved in developmental timing in C. elegans. Genes Dev. 15, 2654-2659 (2001).

18. Lee, Y. S. et al. Distinct roles for Drosophila Dicer-1 and Dicer-2 in the siRNA/miRNA silencing pathways. Cell 117, 69-81 (2004).

19. Matranga, C., Tomari, Y., Shin, C., Bartel, D. P. \& Zamore, P. D. Passenger-strand cleavage facilitates assembly of siRNA into Ago2containing RNAi enzyme complexes. Cell 123, 607-620 (2005).

20. Fukudome, A. et al. Specific requirement of DRB4, a dsRNA-binding protein, for the in vitro dsRNA-cleaving activity of Arabidopsis Dicer-like 4. RNA 17, 750-760 (2011).

21. Nagano, H., Fukudome, A., Hiraguri, A., Moriyama, H. \& Fukuhara, T. Distinct substrate specificities of Arabidopsis DCL3 and DCL4. Nucleic Acids Res. 42, 1845-1856 (2014).

22. Seta, A. et al. Post-translational regulation of the dicing activities of Arabidopsis DICER-LIKE 3 and 4 by inorganic phosphate and the redox state. Plant Cell Physiol. 58, 485-495 (2017).

23. Tabara, M., Ohtani, M., Kanekatsu, M., Moriyama, H. \& Fukuhara, T. Size distribution of small interfering RNAs in various organs at different developmental stages is primarily determined by the dicing activity of Dicer-like proteins in plants. Plant Cell Physiol. 59, 2228-2238 (2018).

24. Blevins, T. et al. Identification of Pol IV and RDR2-dependent precursors of 24 nt siRNAs guiding de novo DNA methylation in Arabidopsis. eLife 4, e09591 (2015).

25. Zhai, J. et al. A one precursor one siRNA model for Pol IV-dependent siRNA biogenesis. Cell 163, 445-455 (2015).

26. Blevins, T. et al. Four plant Dicers mediate viral small RNA biogenesis and DNA virus induced silencing. Nucleic Acids Res. 34, 6233-6246 (2006).

27. Donaire, L. et al. Deep-sequencing of plant viral small RNAs reveals effective and widespread targeting of viral genomes. Virology 392, 203-214 (2009).

28. Zhai, Z., Sooksa-nguan, T. \& Vatamaniuk, O. K. Establishing RNAi as a reverse genetic approach for gene functional analysis in protoplasts. Plant Physiol. 149, 642-652 (2009).

29. Jung, H. I., Zhai, Z. \& Vatamaniuk, O. K. Direct transfer of synthetic double-stranded RNA into protoplasts of Arabidopsis thaliana. Methods Mol. Biol. 744, 109-127 (2011).

30. Niwa, Y., Hirano, T., Yoshimoto, K., Shimizu, M. \& Kobayashi, H. Non-invasive quantitative detection and applications of non-toxic, S65T-type green fluorescent protein in living plants. Plant J. 18, 455-463 (1999).

31. Hong, S. M., Bahn, S. C., Lyu, A., Jung, H. S. \& Ahn, J. H. Identification and testing of superior reference genes for a starting pool of transcript normalization in Arabidopsis. Plant Cell Physiol. 51, 1694-1706 (2010).

32. Dunoyer, P., Himber, C. \& Voinnet, O. DICER-LIKE 4 is required for RNA interference and produces the 21-nucleotide small interfering RNA component of the plant cell-to-cell silencing signal. Nat Genet. 37, 1356-1360 (2005).

33. Henderson, I. R. et al. Dissecting Arabidopsis thaliana DICER function in small RNA processing, gene silencing and DNA methylation patterning. Nat. Genet. 38, 721-725 (2006).

34. Schwarz, D. S. et al. Asymmetry in the assembly of the RNAi enzyme complex. Cell 115, 199-208 (2003).

35. Iki, T. et al. In vitro assembly of plant RNA-induced silencing complexes facilitated by molecular chaperone HSP90. Mol. Cell 39, 282-291 (2010).

36. Mi, S. et al. Sorting of small RNAs into Arabidopsis argonaute complexes is directed by the $5^{\prime}$ terminal nucleotide. Cell $133,116-127$ (2008).

37. Takeda, A., Iwasaki, S., Watanabe, T., Utsumi, M. \& Watanabe, Y. The mechanism selecting the guide strand from small RNA duplexes is different among argonaute proteins. Plant Cell Physiol. 49, 493-500 (2008).

38. Bouché, N., Lauressergues, D., Gasciolli, V. \& Vaucheret, H. An antagonistic function for Arabidopsis DCL2 in development and a new function for DCL4 in generating viral siRNAs. EMBO J. 25, 3347-3356 (2006).

39. Fusaro, A. F. et al. RNA interference-inducing hairpin RNAs in plants act through the viral defence pathway. EMBO Rep. 7 , $1168-1175$ (2006).

40. Xie, Z. et al. Genetic and functional diversification of small RNA pathways in plants. PLoS Biol. 2, e104 (2004). 
41. Pontes, O. et al. The Arabidopsis chromatin-modifying nuclear siRNA pathway involves a nucleolar RNA processing center. Cell 126, 79-92 (2006).

42. Pumplin, N. et al. DNA methylation influences the expression of DICER-LIKE4 isoforms, which encode proteins of alternative localization and function. Plant Cell 28, 2786-2804 (2016).

43. Montavon, T. et al. A specific dsRNA-binding protein complex selectively sequesters endogenous inverted-repeat siRNA precursors and inhibits their processing. Nucleic Acids Res. 45, 1330-1344 (2017).

\title{
Acknowledgements
}

We would like to acknowledge support received from the Gene Research Center at Tokyo University of Agriculture and Technology. This work was supported by the Ministry of Education, Culture, Sports, Science and Technology (MEXT) of Japan (a Grant-in-Aid for Scientific Research [C] [No. 24570044] and Scientific Research on Innovative Areas [Nos 16H06435, $16 \mathrm{H} 06429$ and 16H21723] to T.F.) and the Global Innovation Research (GIR) Organization of Tokyo University of Agriculture and Technology (to T.F.).

\section{Author Contributions}

S.K., M.T., H.M. and T.F. designed the research; S.K., M.T. and Y.N. performed the research; S.K. and M.T. analyzed the data; M.T. and T.F. wrote the article.

\section{Additional Information}

Supplementary information accompanies this paper at https://doi.org/10.1038/s41598-019-43443-9.

Competing Interests: The authors declare no competing interests.

Publisher's note: Springer Nature remains neutral with regard to jurisdictional claims in published maps and institutional affiliations.

\begin{abstract}
(c) (i) Open Access This article is licensed under a Creative Commons Attribution 4.0 International License, which permits use, sharing, adaptation, distribution and reproduction in any medium or format, as long as you give appropriate credit to the original author(s) and the source, provide a link to the Creative Commons license, and indicate if changes were made. The images or other third party material in this article are included in the article's Creative Commons license, unless indicated otherwise in a credit line to the material. If material is not included in the article's Creative Commons license and your intended use is not permitted by statutory regulation or exceeds the permitted use, you will need to obtain permission directly from the copyright holder. To view a copy of this license, visit http://creativecommons.org/licenses/by/4.0/.
\end{abstract}

(C) The Author(s) 2019 